\title{
Review Article \\ The Effects of Virtual Reality Training on Function in Chronic Stroke Patients: A Systematic Review and Meta-Analysis
}

\author{
Han Suk Lee $\mathbb{D}^{1},{ }^{1}$ Yoo Junk Park $\left(\mathbb{D},{ }^{2}\right.$ and Sun Wook Park $\mathbb{D D}^{2}$ \\ ${ }^{1}$ Department of Physical Therapy, Faculty of Health Science, Eulji University, 553 Sanseongdae Ro, Sujeong-gu, Seongnam, \\ Gyeonggi-do 13135, Republic of Korea \\ ${ }^{2}$ Department of Physical Therapy, Samsung Seoul Hospital, 81 Irwon Ro, Gangnam-gu, Seoul 06351, Republic of Korea
}

Correspondence should be addressed to Sun Wook Park; assasun@hanmail.net

Received 12 April 2019; Revised 20 May 2019; Accepted 28 May 2019; Published 18 June 2019

Academic Editor: Matteo Paci

Copyright (C) 2019 Han Suk Lee et al. This is an open access article distributed under the Creative Commons Attribution License, which permits unrestricted use, distribution, and reproduction in any medium, provided the original work is properly cited.

\begin{abstract}
Objective. The aim of this study was to perform a meta-analysis to examine whether virtual reality (VR) training is effective for lower limb function as well as upper limb and overall function in chronic stroke patients. Methods. Three databases, OVID, PubMed, and EMBASE, were used to collect articles. The search terms used were "cerebrovascular accident (CVA)," "stroke", and "virtual reality". Consequently, twenty-one studies were selected in the second screening of meta-analyses. The PEDro scale was used to assess the quality of the selected studies. Results. The total effect size for VR rehabilitation programs was 0.440 . The effect size for upper limb function was 0.431 , for lower limb function it was 0.424 , and for overall function it was 0.545 . The effects of VR programs on specific outcomes were most effective for improving muscle tension, followed by muscle strength, activities of daily living (ADL), joint range of motion, gait, balance, and kinematics. Conclusion. The VR training was effective in improving the function in chronic stroke patients, corresponding to a moderate effect size. Moreover, VR training showed a similar effect for improving lower limb function as it did for upper limb function.
\end{abstract}

\section{Introduction}

Stroke is a major cause of death in the modern world; it also causes sensory, motor, cognitive, and visual impairments and restricts performance of activities of daily living (ADL) [1]. Motor impairments are observed in $80 \%$ of stroke patients, and these can include loss of balance and gait [2]. These problems are important targets of rehabilitation, because they reduce the ability of individuals to perform ADL and this result in impaired community activities $[3,4]$.

Most studies on balance and gait rehabilitation have shown positive effects. However, training-based methods often become tiresome are resource-intensive and require specialized facilities or equipment. Therefore, there is a demand for economical and safe methods of rehabilitation [2].

Virtual reality (VR) is defined by "the use of interactive simulations created with computer hardware and software to present users with opportunities to engage in environments that appear and feel similar to real world objects and events." Participants interact with projected images, maneuver virtual objects and perform activities programmed into the task, giving the user a sense of immersion in the simulated environment. Various forms of feedback are provided through the environment, the most common being visual and auditory, to enhance enjoyment and motor learning through realtime feedback and immediate results [5]. VR training using these features has recently been widely used in the field of stroke rehabilitation [3]. VR training aims to improve neural plasticity by providing a safe and enriched environment to perform functional task-specific activities with increased repetitions, intensity of practice, and motivation to comply with the intervention [1].

In the field of stroke rehabilitation, VR training is reported to be mostly effective at increasing upper limb joint range of motion, improving sensation, muscle strengthening, 
reducing pain, and improving functional processes. Recently, various VR programs have been developed and implemented for the lower limbs as well as the upper limbs, and their effects are being tested. VR training for stroke patients has been shown to be safe and cost-effective at improving lower limb function, specifically improving balance, stair climbing speed, ankle muscle strength, range of motion, and gait speed [1]. Compared with existing treatment methods, it may be more effective at improving dynamic balance control and preventing falls in subacute and chronic stroke patients [6].

Treatment methods using VR provide a virtual environment for ADLs that are difficult to perform in a hospital, and therefore, it could be very effective at improving both upper limb and lower limb function. However, because the lower limbs have to support the weight of the body, various elements are required, including muscle strength and balance to control body weight, joint movements, and cognitive ability to integrate these other elements. Although studies related to VR training have been increasing in recent years, VR intervention has been used more extensively to improve upper limb function, which is relatively easier to apply than lower limb function.

Furthermore, doubts could be raised as to whether VR treatment methods for the lower limbs can improve these elements; these doubts related to lack of VR equipment or programs, as well as safety issues or dizziness during treatment. For this reason, we aimed to perform a metaanalysis as a scientific method to test the effects of uncertain treatment methods using statistical methods, in order to examine whether VR training is effective for lower limb function as well as upper limb function.

\section{Methods}

2.1. Study Search Procedures and Inclusion Criteria. Using the PICOS (Patients, Intervention, Comparison, Outcomes, Study designs) method [7], we investigated patients $(\mathrm{P})$ who had been diagnosed with chronic stroke (more than 6 months after stroke); the intervention (I) was VR rehabilitation therapy, and we compared $(\mathrm{C})$ this with a control group not receiving VR rehabilitation. The outcomes $(\mathrm{O})$ were changes in upper limb function, lower limb function and overall function on daily activity as a primary outcome. Upper limb function was assessed if the chosen instrument measured the impact of upper limb function of individual as FMAUL, muscle strength related to upper limb, JHFT (Jebsen Taylor Hand Function Test), Pinch ability related to hand, Wolf Motor Function Test, ROM related to upper limb, Kinematic data related to upper limb, Grooved Pegboard Test. MAS of upper limb, Motor Activity Log-quality of movement. Lower limb function was assessed if the chosen instrument measured the impact of lower limb function individual as gait variable like speed and cadence, the tools related to balance, Tinetti Performance Oriented Mobility Assessment, FMA-LL, MAS of lower limb, muscle strength test related to lower limb. Overall function was assessed if the chosen instrument measured the impact of total function of individual on every life style like functional independence measure, total FMA, Barthel index score, quality of life.
Study design (S) was randomized control designs. Using OVID, PubMed, and EMBASE, during the same period, two researchers independently searched studies published in English from January 2000 up to June 2018. The search terms used were 'cerebrovascular accident (CVA),' "stroke", and "virtual reality" (Table 1), and discrepancies in the search results were resolved by consensus after a discussion.

A total of 1667 studies were retrieved from the databases after the first search. The inclusion criteria were as follows: studies of patients diagnosed with chronic stroke, studies using VR as a therapeutic intervention, studies that measured function-related changes, and studies that used a randomized controlled trial (RCT) design [8, 9]. The exclusion criteria were graduation theses, books, conference proceedings, single case studies, and quasi-randomized or other qualitative studies. Eventually, 21 articles were included in the metaanalysis based on the PRISMA protocol (Figure 1).

2.2. Quality Assessment. The PEDro scale [31] was used to assess the quality of the selected studies. PEDro scale scores of 9-10 are considered to be of excellent quality, scores of 6-8 points and 4-5 points to be of good and fair quality, respectively, and scores below 4 are of poor quality [32]. The mean PEDro scale score of the selected studies was 6.28 (range from 6 to 8), indicating good quality (Table 2).

2.3. Coding and Data Analysis. For data coding, the authors' names, year of publication, type of publication, research model, study participants, assessment instruments, type of program, and effects of the program were recorded by consensus between a physiotherapist and a meta-analysis expert. Data analysis was performed using CMA 2.0 (Biostat, Englewood, NJ, USA). Heterogeneity was assessed by means of the value of the $\mathrm{I}^{2}$ statistic, where an $\mathrm{I}^{2}$ value greater than $50 \%$ indicated significant heterogeneity. When there was little heterogeneity, pooled analyses were conducted using a fixed effects model. Likewise, when there was great heterogeneity between studies, a random effects model was used [33]. Data were synthesized using a meta-analytic method based on a random effects model due to the small studies even though the results of heterogeneity testing for the studies were not significant $\left(\mathrm{Q}(20)=27.499, \mathrm{I}^{2}=27.269, \mathrm{p}>0.05\right)$ [7]. All effect sizes were changed to the Hedges g statistic because it contains a small sample bias correction [34, 35]. Sensitivity analysis is an analytical method that examines how results change according to the criteria and contents of analysis. We drop the study that score below 4 point in methodological quality of trials from the meta-analysis to confirm its effect on results for sensitivity analysis [7].

\section{Results}

3.1. Data and General Characteristics of the Selected Studies. We used 33 effect sizes from 21 studies. There were 562 patients in total. General information about the studies included in the analysis is shown in Table 3.

3.2. Testing for Publication Bias. In the analysis of publication bias, the funnel plot (Figure 2) was symmetrical, and a trim 
TABLE 1: Strategy for electronic databases survey.

\begin{tabular}{lc}
\hline Electronic databases & Key words (DECS/MeSH) \\
\hline EMBASE & ('cerebrovascular':ab OR 'stroke':ab) AND 'virtual reality':ab $=632$ \\
OVID & $($ (stroke or cerebrovascular) and virtual reality).ab. $=528$ \\
PubMed & (stroke[Title/Abstract] OR cerebrovascular[Title/Abstract]) AND virtual reality[Title/Abstract] $=507$ \\
\hline
\end{tabular}
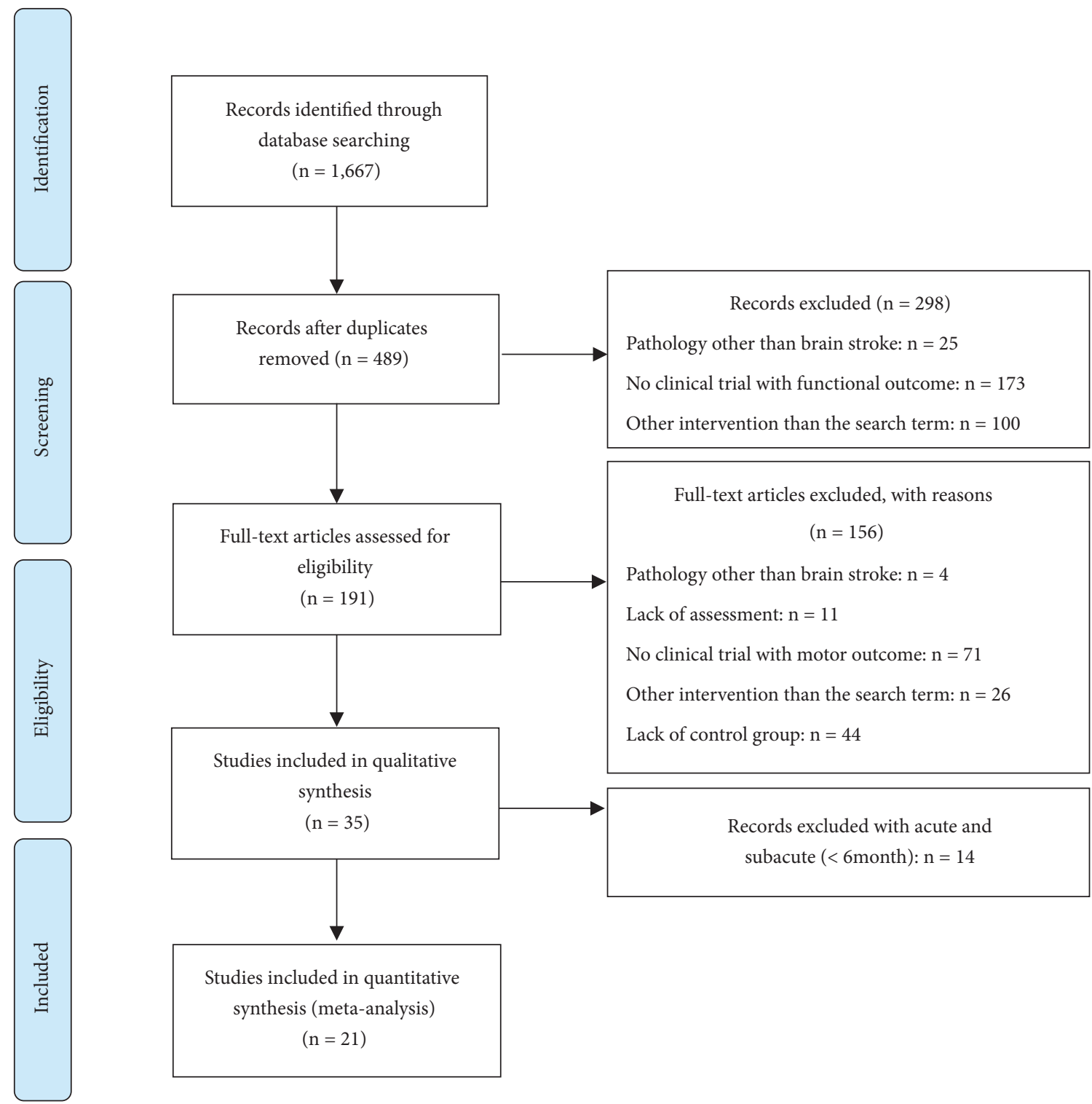

Records excluded with acute and subacute $(<6$ month): $\mathrm{n}=14$

and fill analysis gave a result of 0.440 for both the observed and the adjusted values, $0.440(0.360-0.520)$. When we tested Kendall's tau based on the method by Begg and Mazumdar (1994) [36, 37], there was no significant correlation $(\tau=.281$, $\mathrm{p}>0.05$ ). Therefore, we were able to conclude that the studies in this analysis did not show publication bias.

3.3. Analysis of the Total Effect. To analyze the effects of VR rehabilitation programs, we calculated the effect size as the 'standardized mean difference;' the total effect size for a random effects model was 0.440 , corresponding to a moderate effect size [33], and this was statistically significant $(\mathrm{p}<0.05)(95 \%$ CI: 0.360-520). A forest plot for all 21 studies is shown in Figure 3. Sensitivity analysis showed that the total effect size of the random effects model was 0.446 and the $95 \%$ confidence interval was $0.361-530$, which was statistically significant. As a result of the sensitivity analysis, there is little difference in total effect size except for low quality research. 
TABLE 2: Methodological Quality of Trials $(\mathrm{n}=21)$.

\begin{tabular}{|c|c|c|c|c|c|c|c|c|c|c|c|c|}
\hline Study & score & 1 & 2 & 3 & 4 & 5 & 6 & 7 & 8 & 9 & 10 & 11 \\
\hline Bang (2016) & 4 & 0 & 1 & 0 & 1 & 0 & 0 & 0 & 0 & 0 & 1 & 1 \\
\hline Calabrò (2017) & 9 & 1 & 1 & 1 & 1 & 0 & 1 & 1 & 1 & 1 & 1 & 1 \\
\hline Cho (2012) & 5 & 1 & 1 & 0 & 1 & 0 & 0 & 0 & 1 & 0 & 1 & 1 \\
\hline Cho (2014) & 7 & 0 & 1 & 1 & 1 & 0 & 0 & 1 & 1 & 0 & 1 & 1 \\
\hline da Silva Ribeiro (2015) & 7 & 0 & 1 & 1 & 1 & 0 & 0 & 1 & 1 & 0 & 1 & 1 \\
\hline Fluet (2015) & 4 & 1 & 0 & 0 & 1 & 0 & 0 & 0 & 0 & 1 & 1 & 1 \\
\hline In (2016) & 8 & 1 & 1 & 1 & 1 & 0 & 0 & 1 & 1 & 1 & 1 & 1 \\
\hline Jang (2005) & 5 & 0 & 1 & 0 & 1 & 0 & 0 & 0 & 1 & 0 & 1 & 1 \\
\hline Kim (2015) & 4 & 0 & 1 & 0 & 1 & 0 & 0 & 0 & 0 & 0 & 1 & 1 \\
\hline Kiper (2014) & 5 & 1 & 1 & 0 & 1 & 0 & 0 & 0 & 1 & 0 & 1 & 1 \\
\hline Lee D (2014) & 5 & 1 & 1 & 0 & 1 & 0 & 0 & 1 & 0 & 0 & 1 & 1 \\
\hline Lee (2015) & 5 & 1 & 1 & 0 & 1 & 0 & 0 & 0 & 1 & 0 & 1 & 1 \\
\hline Lee (2016) & 6 & 1 & 1 & 0 & 1 & 0 & 0 & 1 & 1 & 0 & 1 & 1 \\
\hline Llorens (2015) & 8 & 1 & 1 & 1 & 1 & 0 & 0 & 1 & 1 & 1 & 1 & 1 \\
\hline Park (2016) & 8 & 1 & 1 & 1 & 1 & 1 & 0 & 1 & 0 & 1 & 1 & 1 \\
\hline Park (2017) & 6 & 1 & 1 & 1 & 1 & 0 & 0 & 1 & 0 & 0 & 1 & 1 \\
\hline $\operatorname{Sin}(2013)$ & 6 & 1 & 1 & 0 & 1 & 0 & 0 & 1 & 1 & 0 & 1 & 1 \\
\hline Singh (2013) & 9 & 1 & 1 & 1 & 1 & 1 & 0 & 1 & 1 & 1 & 1 & 1 \\
\hline Song (2015) & 3 & 1 & 1 & 0 & 0 & 0 & 0 & 0 & 0 & 0 & 1 & 1 \\
\hline Viana (2014) & 9 & 1 & 1 & 1 & 1 & 1 & 1 & 1 & 1 & 0 & 1 & 1 \\
\hline Yom (2015) & 6 & 1 & 1 & 0 & 1 & 0 & 0 & 1 & 1 & 0 & 1 & 1 \\
\hline
\end{tabular}

PEDro items: 1 Eligibility criteria; 2 Random allocation; 3 Concealed allocation; 4 Baseline Comparability; 5 Blind subjects; 6 Blind therapists; 7 Blind assessors; 8 Adequate follow-up; 9 Intention to treat analysis; 10 Between-group statistical comparisons; 11 Point estimates and variability.

\subsection{The Effects of VR Rehabilitation Programs on Functional} Improvement. The effect size for upper limb function was 0.431 , for lower limb function it was 0.424 , and for overall function it was 0.545 ; these results were statistically significant $(\mathrm{p} \leq 0.001)$ (Table 4$)$. Sensitivity analysis showed that the effect size of the upper limb function was the same as that of the overall function, and the effect size of the lower limb function was 0.432 (CI; 0.339-0.526), which was statistically significant. In other words, there was no significant difference in results except for low quality studies.

3.5. The Effects of VR Rehabilitation Programs on Specific Aspects of Functional Improvement. We compared the effects of VR programs on specific outcomes, and found that, in descending order, it was most effective for improving muscle tension, followed by muscle strength, ADL, joint range of motion, gait, balance, and kinematics. In particular, very large effect sizes were observed for improvements in muscle tension, muscle strength, and ADL. Statistically significant results were observed for all items $(\mathrm{p}<0.05)$ (Table 5).

Sensitivity analysis showed the same results except for balance and gait, but there was no significant difference in balance and gait. In the case of the balance, the effect size of before and after sensitivity analysis were 0.364 (CI: 0.244-0.484) and 0.354 (CI: 0.226-0.482) respectively. In the case of gait, the effect size was 0.445 (CI: 0.309-0.582) before the sensitivity analysis, but the effect size was 0.469 (CI: $0.325-0.613$ ) after the sensitivity analysis.
3.6. Analysis by Program Mode. In a meta-regression analysis by duration of intervention, the slope coefficient was 0.019 (95\% CI: -0.022-0.061), and longer duration was associated with larger effect size; however, the slope coefficient was not statistically significant $(\mathrm{P}>0.05)$. Nevertheless, the slope showed a high effect size as the duration approached 8 weeks (Figure 4). In a meta-regression analysis by weekly frequency of intervention, the slope coefficient was -0.016 (95\% CI: $-0.078-0.046$ ), and as the frequency increased, the effect size decreased; $h$ the slope coefficient was not statistically significant $(\mathrm{P}>0.05)$ (Figure 5).

The sensitivity of the meta-regression analysis was 0.029 for the treatment duration and -0.012 for the frequency per week, which was not statistically significant.

\section{Discussion}

VR treatment methods are economical, provide clear motivation, can improve the effects of treatment, and can provide opportunities for the user to participate in a realistic environment resembling real objects and events by integrating multiple sensory stimuli through visual, auditory, tactile, and somatosensory systems $[1,3,5,14,38]$. For these reasons, VR rehabilitation training has recently emerged as an important method to promote functional recovery after a stroke.

We performed a systematic review and meta-analysis to investigate whether VR rehabilitation training is effective at improving function in chronic stroke patients, and observed moderate effect size $(\mathrm{ES}=0.440)$. Aminov et al. 
TABLE 3: General Characteristics of Included Trials.

\begin{tabular}{|c|c|c|c|c|c|c|}
\hline Study & $\begin{array}{l}\text { Mean } \\
\text { age } \\
\mathrm{E}(\mathrm{C})\end{array}$ & $\begin{array}{l}\text { No. of patients } \\
\text { analyzed } E(C)\end{array}$ & $\begin{array}{l}\text { Months } \\
\text { since } \\
\text { onset } \\
\mathrm{E}(\mathrm{C})\end{array}$ & $\begin{array}{c}\mathrm{VR} \\
\text { Intervention }\end{array}$ & $\begin{array}{l}\text { Control } \\
\text { Intervention }\end{array}$ & Outcome Measures \\
\hline $\begin{array}{l}\text { Bang } \\
(2016) \\
{[10]}\end{array}$ & $\begin{array}{c}62.2 \\
(63.2)\end{array}$ & $\begin{array}{c}20 \\
(20)\end{array}$ & $\begin{array}{l}30.4 \\
(31.6)\end{array}$ & $\begin{array}{l}\text { Wii board balance } \\
\text { system: } \\
\text { yoga, muscular } \\
\text { strength exercise, } \\
\text { aerobic exercise, } \\
\text { balancing exercise } 40 \\
\text { min } * 3 \mathrm{~d} / \mathrm{w} * 8 \text { wks }\end{array}$ & $\begin{array}{l}\text { low-speed treadmills } \\
40 \mathrm{~min} * 3 \mathrm{~d} / \mathrm{w} * 8 \mathrm{wks}\end{array}$ & $\begin{array}{l}\text { Lt/Rt WB } \\
\text { Ant/post WB } \\
\text { Affected side } \\
\text {-stance phase } \\
\text {-swing phase } \\
\text { Cadence }\end{array}$ \\
\hline $\begin{array}{l}\text { Calabrò } \\
(2017) \\
{[11]}\end{array}$ & $\begin{array}{c}60.0 \\
(63.0)\end{array}$ & $\begin{array}{c}12 \\
(12)\end{array}$ & $\begin{array}{c}8.0 \\
(8.0)\end{array}$ & $\begin{array}{c}\mathrm{VR}+\mathrm{RAGT} \text { (robotic } \\
\text { assist gait training) } \\
45 \mathrm{~min} * 5 \mathrm{~d} / \mathrm{w} * 8\end{array}$ & $\begin{array}{l}\text { RAGT (robotic assist } \\
\text { gait training) } \\
45 \mathrm{~min} * 5 \mathrm{~d} / \mathrm{w} * 8\end{array}$ & $\begin{array}{l}\text { RMI } \\
\text { POMA } \\
\text { MAS }\end{array}$ \\
\hline $\begin{array}{l}\text { Cho } \\
(2012) \\
{[12]}\end{array}$ & $\begin{array}{c}65.2 \\
(63.1)\end{array}$ & $\begin{array}{c}11 \\
(11)\end{array}$ & $\begin{array}{l}12.5 \\
(12.6)\end{array}$ & $\begin{array}{l}\text { Nintendo Wii balance } \\
\text { training (balance } \\
\text { Bubble, ski slalom, ski } \\
\text { jump, soccer heading, } \\
\text { table tilting, penguin } \\
\text { slide } 30 \text { min }+ \\
\text { Standard training (PT, } \\
\text { OT) } 30 \text { min } * 5 \mathrm{~d} / \mathrm{w} * 6 \\
\text { wks }\end{array}$ & $\begin{array}{c}\text { Standard training }(\mathrm{PT} \text {, } \\
\text { OT) } 30 \mathrm{~min} * 5 \mathrm{~d} / \mathrm{w} * 6 \\
\text { wks }\end{array}$ & $\begin{array}{l}\text { PSV-apeo } \\
\text { PSV-mleo } \\
\text { PSV-apec } \\
\text { PSV-mlec } \\
\text { BBS / TUG }\end{array}$ \\
\hline $\begin{array}{l}\text { Cho } \\
(2014) \\
{[13]}\end{array}$ & $\begin{array}{c}65.7 \\
(63.5)\end{array}$ & $\begin{array}{l}15 \\
(15)\end{array}$ & $\begin{array}{c}13.8 \\
(15.3)\end{array}$ & $\begin{array}{c}\text { Treadmill training } \\
\text { based real-world video } \\
\text { recording: } 30 \\
\text { min } * 3 \mathrm{~d} / \mathrm{w}, 6 \mathrm{wks}+\mathrm{PT} \\
(\mathrm{NDT}, \mathrm{PNF}) 30 \mathrm{~min} \text {, } \\
\text { OT }(\mathrm{U} / \mathrm{E} \mathrm{ADL}) 30 \mathrm{~min} \text {, } \\
\text { FES } 20 \mathrm{~min} \\
* 5 \mathrm{~d} / \mathrm{w} * 6 \mathrm{wks}\end{array}$ & $\begin{array}{l}\text { Treadmill walking } \\
\text { training: } 30 \mathrm{~min} * 3 \\
\mathrm{~d} / \mathrm{w}, 6 \text { wks }+\mathrm{PT}(\mathrm{NDT}, \\
\mathrm{PNF}) 30 \mathrm{~min}, \mathrm{OT}(\mathrm{U} / \mathrm{E} \\
\mathrm{ADL}) 30 \mathrm{~min}, \mathrm{FES} 20 \\
\mathrm{~min} * 5 \mathrm{~d} / \mathrm{w} * 6 \mathrm{wks}\end{array}$ & $\begin{array}{l}\text { AP, ML-PSV } \\
\text { PSVM } \\
\text { BBS / TUG } \\
\text { Gait speed } \\
\text { Cadence } \\
\text { SLSP, } \%) \text { GC } \\
\text { DLSP,(\%) GC } \\
\text { Step length } \\
\text { Stride length } \\
\end{array}$ \\
\hline $\begin{array}{l}\text { da Silva } \\
\text { Ribeiro } \\
(2015) \\
{[14]}\end{array}$ & $\begin{array}{c}53.7 \\
(52,8)\end{array}$ & $\begin{array}{l}15 \\
(15)\end{array}$ & $\begin{array}{c}42.1 \\
(60.4)\end{array}$ & $\begin{array}{l}\text { Nintendo (tennis, hula } \\
\text { hoop, soccer, boxing } \\
\text { games) } 60 \mathrm{~min} * 2 \\
\text { d/w } * 8 \mathrm{wks}\end{array}$ & $\begin{array}{c}\text { Conventional } \\
\text { therapy(stretching, } \\
\text { trunk and scapular } \\
\text { mobilization, balance, } \\
\text { UL diagonal } \\
\text { movement, gripping, } \\
\text { gait) } 60 \mathrm{~min} * 2 \mathrm{~d} / \mathrm{w} * 8 \\
\text { wks }\end{array}$ & $\begin{array}{l}\text { FMA } \\
\text { SF-36 }\end{array}$ \\
\hline $\begin{array}{l}\text { Fluet } \\
(2015) \\
{[15]}\end{array}$ & $\begin{array}{l}\mathrm{X} \\
(\mathrm{X})\end{array}$ & $\begin{array}{c}10 \\
(11)\end{array}$ & $\begin{array}{c}60.0 \\
(87.0)\end{array}$ & $\begin{array}{c}\text { Robotic/virtually } \\
\text { simulated, } \\
\text { arm and finger } \\
\text { rehabilitation activities: } \\
180 \mathrm{~min} * 4 \mathrm{~d} / \mathrm{w} * 2 \text { wks }\end{array}$ & $\begin{array}{l}\text { Repetitive task practice } \\
\text { on arm and finger } \\
\text { activities: } 180 \mathrm{~min} * 4 \\
\mathrm{~d} / \mathrm{w} * 2 \mathrm{wks}\end{array}$ & $\begin{array}{l}\text { WMFT } \\
\text { FMA-UE } \\
\text { RGT }\end{array}$ \\
\hline $\begin{array}{l}\text { In } \\
(2016) \\
{[16]}\end{array}$ & $\begin{array}{c}57.0 \\
(54.0)\end{array}$ & $\begin{array}{c}13 \\
(12)\end{array}$ & $\begin{array}{l}12.5 \\
(13.6)\end{array}$ & $\begin{array}{c}\text { (VR reflection therapy } \\
\text { 30min + Conventional } \\
\text { therapy: } \\
\text { patient-specific NDT, } \\
\text { PT, OT, ST } 30 \mathrm{~min}) * 5 \\
\text { d/w*4wks }\end{array}$ & $\begin{array}{c}\text { (Placebo VR 30min } * \\
5 \mathrm{~d} / \mathrm{w} * 4 \text { wks } 30 \mathrm{~min}+ \\
\text { Conventional therapy: } \\
\text { patient-specific NDT, } \\
\text { PT, OT, ST } \\
\text { 30min) } * 5 \mathrm{~d} / \mathrm{w} * 4 \mathrm{wks} \\
\end{array}$ & $\begin{array}{l}\text { BBS / FRTTUG } \\
\text { EO APS, MLS, } \\
\text { TSEC APS, MLS, } \\
\text { TS10mWV }\end{array}$ \\
\hline $\begin{array}{l}\text { Jang } \\
(2005) \\
{[17]}\end{array}$ & $\begin{array}{c}59.8 \\
(54.4)\end{array}$ & $\begin{array}{c}5 \\
(5)\end{array}$ & $\begin{array}{l}13.8 \\
(13.4)\end{array}$ & $\begin{array}{l}\text { VR(reaching, lifting, } \\
\text { and grasping motor } \\
\text { skills game) } \\
60 \mathrm{~min} * 5 \mathrm{~d} / \mathrm{w} * 4 \mathrm{wks}\end{array}$ & No treatment & $\begin{array}{c}\text { BBT } \\
\text { MFT } \\
\text { FMA-UE }\end{array}$ \\
\hline
\end{tabular}


TABle 3: Continued.

\begin{tabular}{|c|c|c|c|c|c|c|}
\hline Study & $\begin{array}{l}\text { Mean } \\
\text { age } \\
\mathrm{E}(\mathrm{C})\end{array}$ & $\begin{array}{l}\text { No. of patients } \\
\text { analyzed } E(C)\end{array}$ & $\begin{array}{c}\text { Months } \\
\text { since } \\
\text { onset } \\
\mathrm{E}(\mathrm{C})\end{array}$ & $\begin{array}{c}\mathrm{VR} \\
\text { Intervention }\end{array}$ & $\begin{array}{c}\text { Control } \\
\text { Intervention }\end{array}$ & Outcome Measures \\
\hline $\begin{array}{l}\text { Kim } \\
(2015) \\
{[18]}\end{array}$ & $\begin{array}{c}\mathrm{X} \\
(\mathrm{X})\end{array}$ & $\begin{array}{l}10 \\
(7)\end{array}$ & $>6$ & $\begin{array}{l}\text { Community-based } \\
\text { virtual reality scene } \\
\text { exposure combined } \\
\text { with treadmill training: } \\
30 \mathrm{~min} * 3 \mathrm{~d} / \mathrm{w} * 4 \mathrm{wks}+ \\
\text { Conventional } \mathrm{PT} \\
\text { (muscle strengthening, } \\
\text { balance training, and } \\
\text { indoor and outdoor } \\
\text { gait training): } 60 \\
\text { min } * 5 \mathrm{~d} / \mathrm{w} * 4 \mathrm{wks}\end{array}$ & $\begin{array}{l}\text { Over ground walking, } \\
\text { stair walking, slope } \\
\text { walking, and unstable } \\
\text { surface walking for } 570 \\
\mathrm{~m} 30 \mathrm{~min} * 3 \mathrm{~d} / \mathrm{w} * 4 \\
\text { wks + Conventional } \\
\text { PT(muscle } \\
\text { strengthening, balance } \\
\text { training, and indoor } \\
\text { and outdoor gait } \\
\text { training): } 60 \text { min } * 5 \\
\text { d/w } * 4 \mathrm{wks}\end{array}$ & $\begin{array}{l}\text { PSPL AP } \\
\text { PSPL ML } \\
\text { PSPL total } \\
\text { APSS }\end{array}$ \\
\hline $\begin{array}{l}\text { Kiper } \\
(2014) \\
{[19]}\end{array}$ & $\begin{array}{c}63.1 \\
(65.5)\end{array}$ & $\begin{array}{c}23 \\
(21)\end{array}$ & $>12$ & $\begin{array}{l}\text { Reinforced feedback in } \\
\text { virtual environment: } \\
\text { grasping and reaching } \\
\text { movement } 120 \mathrm{~min} * 5 \\
\mathrm{~d} / \mathrm{w} * 4 \mathrm{wks}\end{array}$ & $\begin{array}{l}\text { Exercises of various } \\
\text { movements in a } \\
\text { horizontal or vertical } \\
\text { plane } 120 \mathrm{~min} * 5 \\
\mathrm{~d} / \mathrm{w} * 4 \mathrm{wks}\end{array}$ & $\begin{array}{c}\text { FMA-UE } \\
\text { FIM } \\
\text { Movement } \\
\text {-Time }(\mathrm{sec}) \\
\text {-Speed }(\mathrm{cm} / \mathrm{sec}) \\
\text {-Peak }(\mathrm{n}) \\
\end{array}$ \\
\hline $\begin{array}{l}\text { Lee D } \\
(2014) \\
{[20]}\end{array}$ & $\begin{array}{c}58.3 \\
(65.4)\end{array}$ & $\begin{array}{c}12 \\
(12)\end{array}$ & $\begin{array}{c}9.3 \\
(8.9)\end{array}$ & $\begin{array}{c}\text { VRRE (Asymmetric } \\
\text { training on hand): } 30 \\
\text { min } * 5 \mathrm{~d} / \mathrm{w} * 4 \text { wks }+ \\
\text { Standard rehabilitation } \\
\text { training: gait, } \\
\text { strengthening } 60 \\
\text { min } * 5 \mathrm{~d} / \mathrm{w} * 4 \mathrm{wks}+ \\
\text { FES } 25 \mathrm{~m} \text { in } * 5 \mathrm{~d} / \mathrm{w} * 4 \\
\text { wks }\end{array}$ & $\begin{array}{c}\text { Symmetric training on } \\
\text { hand: } 30 \mathrm{~min} * 5 \mathrm{~d} / \mathrm{w} * \\
4 \mathrm{wks}+\text { Standard } \\
\text { rehabilitation training: } \\
\text { NDT(gait, } \\
\text { strengthening): } 60 \\
\min * 5 \mathrm{~d} / \mathrm{w} * 4 \mathrm{wks}+ \\
\text { FES } 25 \mathrm{~min} * * 5 \mathrm{~d} / \mathrm{w} * 4 \\
\text { wks }\end{array}$ & $\begin{array}{c}\text { FMA-UE } \\
\text { BBT } \\
\text { Grip strength } \\
\text { MAS-UE }\end{array}$ \\
\hline $\begin{array}{l}\text { Lee H } \\
(2015) \\
{[21]}\end{array}$ & $\begin{array}{c}45.9 \\
(49.2)\end{array}$ & $\begin{array}{c}12 \\
(12)\end{array}$ & $>6$ & $\begin{array}{l}\text { VR training(sitting } \\
\text { posture, knee bend \& } \\
\text { other leg knee extend, } \\
\text { tightrope walking, } \\
\text { penguin teeter-totter } \\
\text { seesaw, balance skiing, } \\
\text { rolling marble board, } \\
\text { balance Wii) } 30 \text { min } * 3 \\
\text { d/w*6 wks }+ \text { General } \\
\text { exercise } 60 \text { min } * 5 \\
\text { d/w } * 6 \text { wks }\end{array}$ & $\begin{array}{l}\text { Task-oriented training } \\
\text { (sit to stand from } \\
\text { different heights, task } \\
\text { training in standing, } \\
\text { balance training on an } \\
\text { unstable surface, lifting } \\
\text { a leg in place, kicking a } \\
\text { ball, stair climbing and } \\
\text { descending) } 30 \text { min } * 3 \\
\text { d/w*6 wks }+ \text { General } \\
\text { exercise } 60 \text { min } * 5 \\
\text { d/w } 66 \text { wks }\end{array}$ & $\begin{array}{c}\text { EOWB COP } \\
\text { ECWB COP } \\
\text { EONB COP } \\
\text { ECNB COP } \\
\text {-Path length, } \\
\text {-velocity } \\
\text { FRT }\end{array}$ \\
\hline $\begin{array}{l}\text { Lee S } \\
(2016) \\
{[22]}\end{array}$ & $\begin{array}{c}69.2 \\
(73.1)\end{array}$ & $\begin{array}{l}10 \\
(8)\end{array}$ & $\begin{array}{c}16.2 \\
(17.0)\end{array}$ & $\begin{array}{c}\text { Virtual reality-based } \\
\text { bilateral upper } \\
\text { extremity training } \\
30 \mathrm{~min} * 3 \mathrm{~d} / \mathrm{w} * 6 \mathrm{wks}+ \\
\text { Conventional OT } \\
30 \mathrm{~min} * 5 \mathrm{~d} / \mathrm{w} * 6 \mathrm{wks}\end{array}$ & $\begin{array}{l}\text { Watching an irrelevant } \\
\text { video in a VR } \\
\text { environment with } \\
\text { bilateral upper } \\
\text { extremity training } 30 \\
\text { min } * 3 \mathrm{~d} / \mathrm{w} * 6 \text { wks }+ \\
\text { Conventional OT } 30 \\
\text { min } * 5 \mathrm{~d} / \mathrm{w} * 6 \text { wks }\end{array}$ & $\begin{array}{c}\text { JHFT } \\
\text { BBT } \\
\text { GPT } \\
\text { Strength } \\
\text {-biceps, triceps } \\
\text { grip strength } \\
\text { palmar pinch } \\
\text { lateral pinch } \\
\text { tip pinch }\end{array}$ \\
\hline
\end{tabular}


TABle 3: Continued.

\begin{tabular}{|c|c|c|c|c|c|c|}
\hline Study & $\begin{array}{l}\text { Mean } \\
\text { age } \\
\mathrm{E}(\mathrm{C})\end{array}$ & $\begin{array}{l}\text { No. of patients } \\
\text { analyzed } E(C)\end{array}$ & $\begin{array}{l}\text { Months } \\
\text { since } \\
\text { onset } \\
\mathrm{E}(\mathrm{C}) \\
\end{array}$ & $\begin{array}{c}\text { VR } \\
\text { Intervention }\end{array}$ & $\begin{array}{c}\text { Control } \\
\text { Intervention }\end{array}$ & Outcome Measures \\
\hline $\begin{array}{l}\text { Llorens } \\
(2015) \\
{[23]}\end{array}$ & $\begin{array}{c}58.3 \\
(55.0)\end{array}$ & $\begin{array}{c}10 \\
(10)\end{array}$ & $\begin{array}{c}13.6 \\
(19.6)\end{array}$ & $\begin{array}{l}\text { (Audiovisual feedback } \\
\text { while performing a } \\
\text { stepping task } 30 \mathrm{~min}+ \\
\text { Conventional therapy } \\
30 \mathrm{~min}) * 5 \mathrm{~d} / \mathrm{w} * 4 \mathrm{wks}\end{array}$ & $\begin{array}{l}\text { Conventional therapy } \\
\text { (static standing } \\
\text { exercises in different } \\
\text { positions, task-specific } \\
\text { reaching exercises, } \\
\text { stepping tasks, static } \\
\text { and dynamic balance } \\
\text { exercises, walking } \\
\text { exercises } \\
\text { under different } \\
\text { conditions) } 60 \mathrm{~min} * 5 \\
\mathrm{~d} / \mathrm{w}, * 4 \mathrm{wks}\end{array}$ & $\begin{array}{c}\text { BBS } \\
\text { POMA-balance } \\
\text { POMA-gait } \\
\text { 10-m walking }\end{array}$ \\
\hline $\begin{array}{l}\text { Park } \\
(2016) \\
{[24]}\end{array}$ & $\begin{array}{c}61.6 \\
(62.0)\end{array}$ & $\begin{array}{l}15 \\
(15)\end{array}$ & $>6$ & $\begin{array}{l}\text { Wii Sports \& Resort: } \\
\text { bowling, table tennis, } \\
\text { and canoeing } 30 \mathrm{~min} * \\
5 \mathrm{~d} / \mathrm{w} * 4 \text { wks }+ \text { mental } \\
\text { practice } 5 \text { min } * 5 \\
\text { d/w } * 4 \text { wks }\end{array}$ & $\begin{array}{l}\text { Wii Sports and Sports } \\
\text { Resort games: bowling, } \\
\text { table tennis, and } \\
\text { canoeing games } 30 \\
\text { min } * 5 \mathrm{~d} / \mathrm{w} * 4 \mathrm{wks}\end{array}$ & $\begin{array}{c}\text { FMA-UE } \\
\text { BBT } \\
\text { MAL-QOM }\end{array}$ \\
\hline $\begin{array}{l}\text { Park } \\
(2017) \\
{[25]}\end{array}$ & $\begin{array}{c}62.0 \\
(65.3)\end{array}$ & $\begin{array}{c}10 \\
(10)\end{array}$ & $\begin{array}{c}10.8 \\
(14.1)\end{array}$ & $\begin{array}{l}\text { Xbox Kinect (Boxing, } \\
\text { Table tennis, Soccer, } \\
\text { Golf, Ski, Football) } 30 \\
\text { min + Conventional PT } \\
30 \text { min } * 5 \text { d/w, } 6 \text { wks }\end{array}$ & $\begin{array}{c}\text { Conventional } \\
\text { PT(NDT, PNF) } 30 \text { min } \\
* 5 \mathrm{~d} / \mathrm{w} * 6 \text { wks }\end{array}$ & $\begin{array}{c}\text { FMA-LE } \\
\text { BBS } \\
\text { TUG } \\
\text { 10MWT }\end{array}$ \\
\hline $\begin{array}{l}\text { Sin } \\
(2013) \\
{[26]}\end{array}$ & $\begin{array}{c}71.8 \\
(75.6)\end{array}$ & $\begin{array}{c}18 \\
(17)\end{array}$ & $\begin{array}{c}7.2 \\
(8.5)\end{array}$ & $\begin{array}{c}\text { (Xbox Kinect sports } \\
\text { and adventure } 30 \mathrm{~min} \\
+ \text { standard training } 30 \\
\text { min }) * 3 / \mathrm{w} * 6 \mathrm{wks}\end{array}$ & $\begin{array}{l}\text { standard training: } 30 \\
\min * 3 \mathrm{~d} / \mathrm{w} * 6 \mathrm{wks}\end{array}$ & $\begin{array}{l}\text { ROM-UE } \\
\text { FMA-UE } \\
\text { BBT }\end{array}$ \\
\hline $\begin{array}{l}\text { Singh } \\
(2013) \\
{[27]}\end{array}$ & $\begin{array}{c}65.4 \\
(67.0)\end{array}$ & $\begin{array}{c}15 \\
(13)\end{array}$ & $\begin{array}{c}40.5 \\
(34.9)\end{array}$ & $\begin{array}{l}\text { (Nintendo Balance: } \\
\text { Bubble, Xbox } 360 \\
\text { Kinect: Rally Ball } \\
\text { 30min + standard PT) } \\
90 \mathrm{~min} * 2 \mathrm{~d} / \mathrm{w} * 6 \mathrm{wks}\end{array}$ & $\begin{array}{c}\text { Standard PT } \\
\text { (self-stretching and } \\
\text { strengthening } \\
\text { exercises, coordination } \\
\text { and balance exercises, } \\
\text { functional exercises) } \\
120 \mathrm{~min} * 2 \mathrm{~d} / \mathrm{w} * 6 \mathrm{wks}\end{array}$ & $\begin{array}{c}\text { BI } \\
\text { OBS } \\
6 \mathrm{MWT} \\
\text { 30sSTS } \\
\text { TUG } \\
\text { T10mWT }\end{array}$ \\
\hline $\begin{array}{l}\text { Song } \\
(2015) \\
{[28]}\end{array}$ & $\begin{array}{c}51.4 \\
(50.1)\end{array}$ & $\begin{array}{c}20 \\
(20)\end{array}$ & $\begin{array}{l}14.8 \\
(14.3)\end{array}$ & $\begin{array}{c}\text { Xbox Kinect Sport, } \\
\text { Sport Season 2, } \\
\text { Adventure, and Kinect } \\
\text { Gunstringer } 30 \text { min } * 5 \\
\text { d/w*8 wks }\end{array}$ & $\begin{array}{l}\text { ergometer training } 30 \\
\text { min } * 5 \mathrm{~d} / \mathrm{w} * 8 \text { wks }\end{array}$ & $\begin{array}{c}\text { Affected side WB } \\
\text { Forward LOS } \\
\text { Backward LOS } \\
\text { TUG } \\
\text { 10MWT } \\
\end{array}$ \\
\hline $\begin{array}{l}\text { Viana } \\
(2014) \\
{[29]}\end{array}$ & $\begin{array}{c}56.0 \\
(55.0)\end{array}$ & $\begin{array}{c}10 \\
(10)\end{array}$ & $\begin{array}{c}31.9 \\
(35.0)\end{array}$ & $\begin{array}{l}\text { VR exercises for the } \\
\text { UL (Wii Sports resort, } \\
\text { Wii Play Motion, Let's } \\
\text { Tap) } 60 \mathrm{~min} * 3 \mathrm{~d} / \mathrm{w} * 5 \\
\text { wks + tDCS (primary } \\
\text { motor cortex) } 13 \\
\text { min } * 3 \mathrm{~d} / \mathrm{w} * 5 \text { wks }\end{array}$ & $\begin{array}{l}\text { VR exercises for the } \\
\text { UL(Wii Sports resort, } \\
\text { Wii Play Motion, Let's } \\
\text { Tap) } 60 \mathrm{~min} * 3 \mathrm{~d} / \mathrm{w} * 5 \\
\text { wks }+ \text { sham tDCS } \\
\text { (primary motor } \\
\text { cortex) } 13 \mathrm{~min} * 3 \\
\mathrm{~d} / \mathrm{w} * 5 \mathrm{wks}\end{array}$ & $\begin{array}{c}\text { FMA-UL } \\
\text { WMFT-time } \\
\text { WMFT-FAS } \\
\text { MAS } \\
\text { Grip strength } \\
\text { SSQOL } \\
\text { SSQOL -UL\% }\end{array}$ \\
\hline
\end{tabular}


TABLE 3: Continued.

\begin{tabular}{|c|c|c|c|c|c|c|}
\hline Study & $\begin{array}{l}\text { Mean } \\
\text { age } \\
\mathrm{E}(\mathrm{C})\end{array}$ & $\begin{array}{l}\text { No. of patients } \\
\text { analyzed } E(C)\end{array}$ & $\begin{array}{l}\text { Months } \\
\text { since } \\
\text { onset } \\
\mathrm{E}(\mathrm{C})\end{array}$ & $\begin{array}{c}\text { VR } \\
\text { Intervention }\end{array}$ & $\begin{array}{c}\text { Control } \\
\text { Intervention }\end{array}$ & Outcome Measures \\
\hline $\begin{array}{l}\text { Yom } \\
(2015) \\
{[30]}\end{array}$ & $\begin{array}{l}64.6 \\
(78.1)\end{array}$ & $\begin{array}{l}10 \\
(10)\end{array}$ & $\begin{array}{c}11.1 \\
(11.6)\end{array}$ & $\begin{array}{c}\text { Virtual reality-based } \\
\text { ankle exercise (VRAE): } \\
\text { exercising on the floor, } \\
\text { balance board, cushion } \\
\text { ball, standing on one } \\
\text { foot. } 30 \mathrm{~min} * 5 \mathrm{~d} / \mathrm{w} * 6 \\
\text { wks + Conventional } \\
\text { PT } 60 \mathrm{~min} * 5 \mathrm{~d} / \mathrm{w} * 6 \\
\text { wks }\end{array}$ & $\begin{array}{c}\text { Watching an } \\
\text { environmental } \\
\text { documentary } \\
\text { irrelevant to ankle } \\
\text { exercise } 30 \text { min } * 5 \\
\text { d/w } 6 \text { wks }+ \\
\text { Conventional PT } 60 \\
\text { min } * 5 \mathrm{~d} / \mathrm{w} * 6 \text { wks }\end{array}$ & $\begin{array}{c}\text { MAS } \\
\text { Tardieu scale } \\
\text { TUG } \\
\text { Velocity } \\
\text { Cadence } \\
\text { Step length } \\
\text { Stride length } \\
\text { Stance time \% } \\
\text { Swing time \% } \\
\text { Double limb } \\
\text { support }\end{array}$ \\
\hline
\end{tabular}

RMI (Rivermead mobility index), MAS (modified Ashworth scale), PSV-apeo (postural sway velocity-AP eye open), PSV-mlec (postural sway velocity-ML eye close), BBS (Berg Balance Scale), TUG (Timed up and go test), AP-PSV (anteroposterior postural sway velocity), ML-PSV (mediolateral postural sway velocity), PSVM (postural sway velocity moment), SLSP (single limb support period), GC (gait cycle), DLSP (double limb support period), FMA (Fugl Myer Assessment), SF-36 (Short Form 36 Health Survey), WMFT (Wolf Motor Function Test), RGT (Reach to Grasp Test), FRT (functional reach test), EO-APS (eyes open anterior-posterior sway distance), EO-MLS (eyes open medial-lateral sway distance), EO-TS (eyes open total sway distance), EC-APS (eyes closed anterior-posterior sway distance), EC-MLS (eyes closed medial-lateral sway distance), EC-TS (eyes closed total sway distance), 10-m WV (10-m walking velocity), 10-m WV (10-m walking velocity), BBT (Box and Block Test), MFT (manual function test), PSP L (postural sway path length), PSPL (postural sway path length), PSPL (postural sway path length), APSS (average postural sway speed), FIM (functional independence measure), ECWB COP (eyes-closed wide base), EONB COP (eyes-open narrow base), FRT (functional reach test), JHFT (Jebsen-Taylor hand function test), GPT (grooved pegboard test), MAL-QOM (motor activity log-quality of movement), BI (Barthel index), OBS (Overall balance score), 6MWT (six-minute walk test), 30-s STS (thirty-second sit to stand test), T10-m WT (timed ten-meter walk test), LOS (limit of stability), WMFT (wolf motor function test), WMFT-FAS (functional ability), SSQOL-UL (stroke specific quality of life-upper limb).

TABLE 4: Effect size of Virtual Reality Based Rehabilitation Program on function.

\begin{tabular}{lccccc}
\hline Function & K & ES & SE & p-value & 95\% CI \\
\hline Upper Limb Function & 53 & 0.431 & 0.054 & 0.001 & $0.424-0.537$ \\
\hline Lower Limb Function & 74 & 0.424 & 0.045 & 0.001 & $0.336-0.513$ \\
\hline Overall Function & 6 & 0.545 & 0.149 & 0.001 & $0.253-0.837$ \\
\hline
\end{tabular}

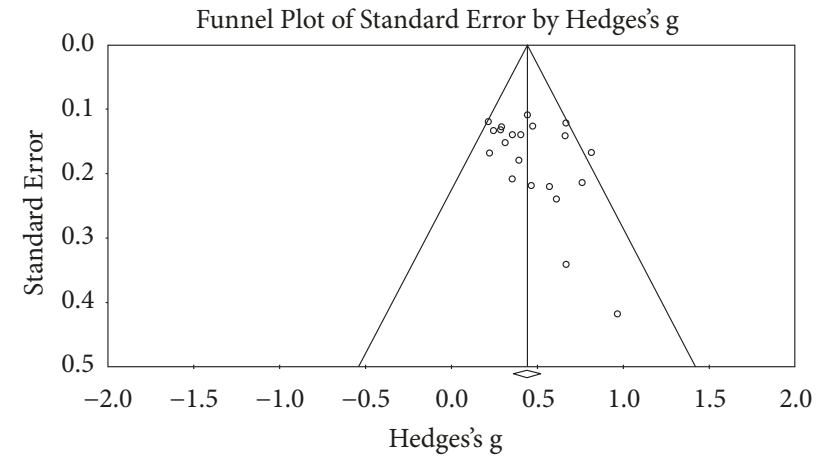

FIGURE 2: Funnel plot for publication bias.

[39] reported that VR provides additional benefits compared to conventional methods, and that it can bring immediate and long-term improvement in post-stroke motor function. We also observed moderate effect size for VR rehabilitation training, suggesting that this technique may be used as a complementary treatment method alongside traditional rehabilitation therapy.

When we performed a subgroup analysis of the effects on functional improvement, the effect size was moderate for upper limb function (ES $=0.431$ ), lower limb function $(\mathrm{ES}=0.424)$, and overall function $(\mathrm{ES}=0.545)$. Therefore, VR rehabilitation training improved lower limb function, including balance and gait, to a similar degree as upper limb function in chronic stroke patients, and it also improved overall physical function. Previous studies reported that use of VR in chronic stroke patients produced significant improvements in functional balance, gait velocity, cadence, and stride length [1], and that performing VR training alongside balance and gait treatment was more effective for improving gait speed and TUG than balance and gait training alone [2]. Other meta-analyses suggested that VR training improves BBS and TUG in chronic stroke patients compared to conventional rehabilitation [3, 40]. Chen et al. [6] provided moderate evidence to support the claim that VR training is effective as a complementary therapy to a standard rehabilitation program to improve balance in chronic stroke patients; however the effects were unclear in acute or subacute stroke patients. In a systematic review, Moreira et al. [4] claimed that training using VR had potential as a method to improve gait parameters in chronic stroke patients, irrespective of the number of participants in the study, participant characteristics, and protocol diversity. Similarly, we found that, although VR programs can cause some discomfort, including dizziness 
TABLE 5: Effect size of Virtual Reality Based Rehabilitation Program on outcomes.

\begin{tabular}{|c|c|c|c|c|c|}
\hline Outcomes & $\mathrm{K}$ & ES & SE & p-value & $95 \% \mathrm{CI}$ \\
\hline Muscle tone & 5 & 0.755 & 0.196 & 0.001 & $0.372-1.139$ \\
\hline Muscle strength & 7 & 0.750 & 0.177 & 0.001 & $0.403-1.098$ \\
\hline $\mathrm{ADL}$ & 3 & 0.627 & 0.199 & 0.002 & $0.237-1.018$ \\
\hline $\mathrm{ROM}$ & 10 & 0.517 & 0.114 & 0.001 & $0.294-0.741$ \\
\hline Gait & 30 & 0.445 & 0.070 & 0.001 & $0.309-0.582$ \\
\hline Function & 23 & 0.388 & 0.085 & 0.001 & $0.222-0.554$ \\
\hline Balance & 40 & 0.364 & 0.061 & 0.001 & $0.244-0.484$ \\
\hline Kinematics & 12 & 0.274 & 0.109 & 0.012 & $0.060-0.488$ \\
\hline
\end{tabular}

Study name

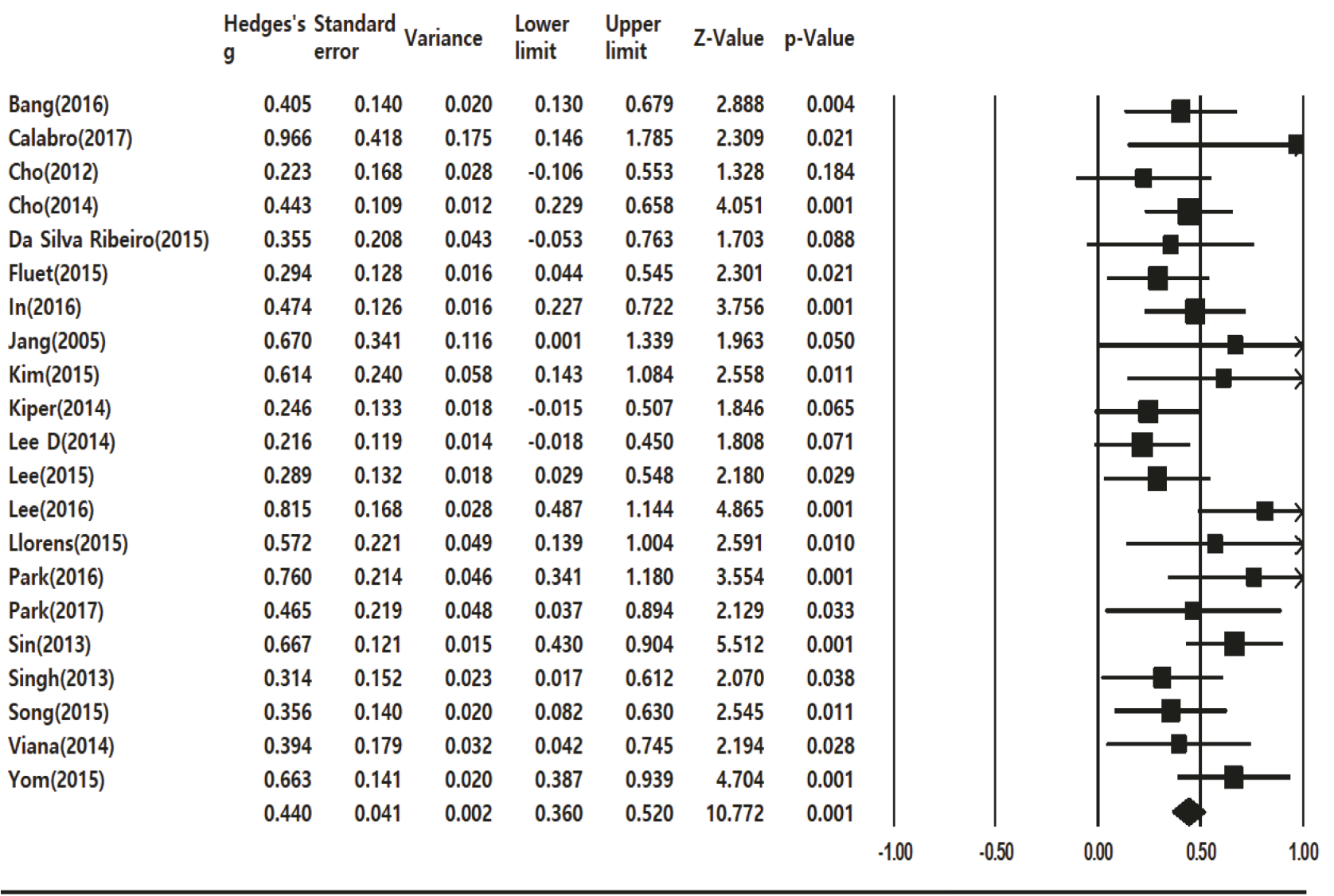

Figure 3: Effect size of Virtual Reality-Based Rehabilitation Program.

[41], they are as effective at improving lower limb function as they are at improving upper limb function. This is thought to be because most programs included in the analysis consisted of game-based tasks, using equipment such as the Nintendo Wii or Xbox Kinect, meaning that subjects had little difficulty using the programs. Programs in the form of games have similar beneficial effects on upper limb function, lower limb function, and overall function.
Laver et al. [42] reported that VR and interactive video games were no better at improving upper limb function in stroke patients than was conventional treatment, and that their effects on gait speed, balance, participation, and quality of life were also unclear. Nevertheless, implementing VR training together with conventional treatment significantly improved upper limb function and helped improve ADLs in a similar way to extending the overall treatment duration. Most 


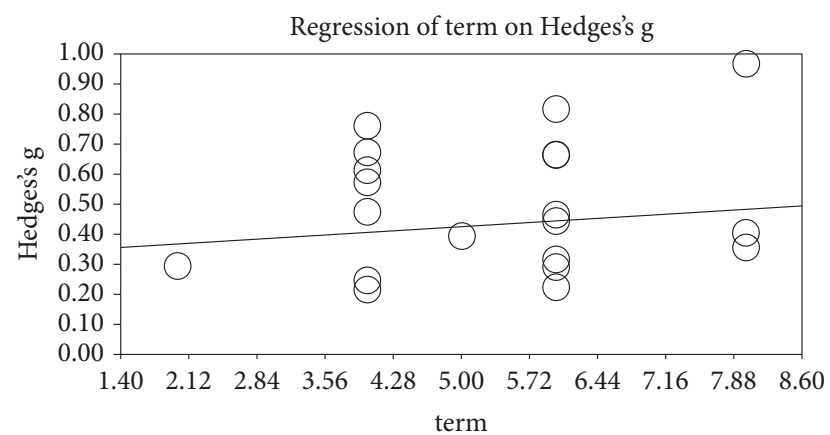

FIGURE 4: Results of the meta-regression analysis by intervention duration (weeks).

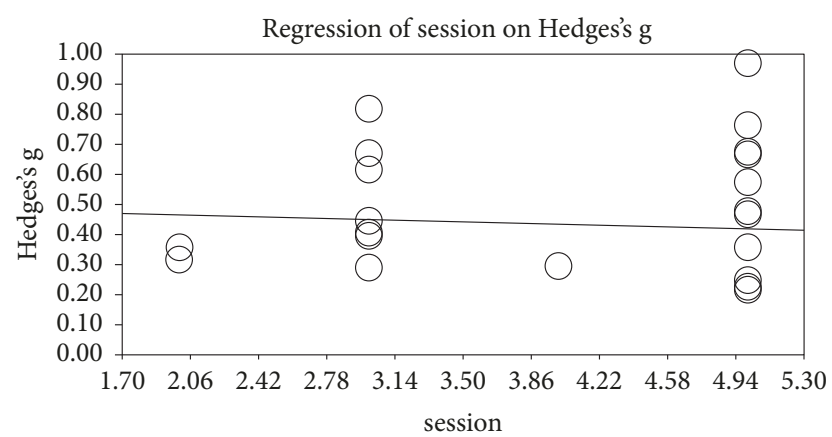

FIGURE 5: Results of the meta-regression analysis by weekly intervention frequency.

of the studies in our analysis also increased the overall treatment time by adding VR training to conventional treatment, and it is possible that this is the reason we observed effects for both the upper and lower limbs, despite most studies also using game-based programs.

When we investigated the effects of VR rehabilitation training on specific aspects of function in chronic stroke patients, we observed relatively strong effects on muscle tension $(\mathrm{ES}=0.755)$ and muscle strength $(\mathrm{ES}=0.722)$, but only moderate to relatively weak effects on (in descending order of effect size) ADLs, joint range of motion, gait, function, balance, and kinematics ( $E S=0.627-0.274)$. Lee [43] reported that voluntary mobility training using Xbox Kinect improved muscle strength, resulting in improved ability to perform ADLs. In a meta-analysis by Laver et al. [42], VR was found to have a moderate size effect at improving ADLs. Meanwhile, in another systematic report and meta-analysis, Aminov et al. [39] reported that VR training had significant effects on body structure/function and activity level as a complementary measure to conventional treatment methods for post-stroke rehabilitation.

Like previous studies, we also found that VR training could be very helpful for improving muscle strength and ADL. This is thought to be because VR training can enhance high-intensity, repeated, task-oriented training typically used as an evidence-based intervention method in chronic stroke patients.

When we analyzed the effects of VR training by treatment duration, a longer duration was associated with a stronger effect, and analysis of the slope showed that a duration closer to 8 weeks resulted in a strong effect. However, it was not statistically significant $(\mathrm{p}=0.364)$.

Also, in the meta-regression analysis by weekly treatment frequency, a higher frequency was actually associated with a reduced effect. However, it was not statistically significant $(\mathrm{p}=0.614)$.

Given that physical adaptation to exercise usually occurs after 6-8 weeks [44], our results suggest that duration of at least 8 weeks is required to obtain an effect from VR. However, most of the studies included in our analysis had a duration of 6 weeks or less, and there were only three studies with a duration over 6 weeks. We believe that this is because the characteristics of chronic stroke patients make longterm VR treatment difficult. Nevertheless, researchers should consider the fact that a duration of at least 8 weeks is required for physical adaptation. In other words, it seems that there was not a significant difference in treatment effect because it took some time to adapt to VR treatment in patients with chronic phase. However, since it is not statistically significant, it will be necessary to conduct additional analysis on more research items in the future.

While the treatment duration of most of the included studies was short, there were many studies that increased the weekly treatment frequency. Nevertheless, the metaregression analysis showed that the studies that increased frequency showed a smaller effect than the studies with lower treatment frequencies. In particular, there was a slight decrease in cases of more than four times. This demonstrates that chronic stroke patients require rest to learn movements. However, the number of studies included in this study was limited and the results were not statistically significant. Therefore, it is considered that there is a limit to interpretation, and it will be necessary to reanalyze more researches in future.

This study had several limitations. First, the lack of studies caused some difficulties performing a subgroup analysis. This is thought to be because we restricted the years of publication and only used three databases. For this reason, further meta-analysis will be required. Second, the type of VR program could also affect the therapeutic effect; however, the intervention types in the studies included in our analysis were mostly game programs, and there were limitations in categorizing these. Therefore, if more diverse training programs are developed in the future, it will be necessary to perform an analysis by program type. Third, even though VR training has been demonstrated to be effective at improving ADLs, because the number of studies included in the analysis was very small, there are limitations in generalizing the results. There have been very few researchers who have measured ADLs, and therefore, in the future, we recommend that researchers plan to study VR by investigating the effects of VR on ADLs.

\section{Conclusions}

This was a systematic review and meta-analysis to examine whether VR rehabilitation training helps to improve function 
in chronic stroke patients, and the results showed a moderatesized effect. Moreover, VR rehabilitation training showed a similar effect for improving lower limb function as it did for upper limb function. Finally, we verified that VR training requires a duration of at least 8 weeks, and that occasional treatment is actually more effective than treatment every day.

This study has a moderate evidence to support the effect of VR on lower extremity function in patients with chronic stroke. Therefore, VR training would be helpful in improving functional outcomes with chronic stroke patients such as gait (speed, cadence, 10MWT, 6MWT), balance (BBS, TUG, postural sway), lower limb movement (FMA, RMI), lower limb strength, and lower limb muscle tone. However, the details on how to use VR program must be set according to the therapeutic goals.

\section{Conflicts of Interest}

The authors declare that there are no conflicts of interest regarding the publication of this paper.

\section{Acknowledgments}

This study was funded by Grant No. EJBS 18-05 from Eulji University in 2018.

\section{References}

[1] E. M. Gibbons, A. Nicole Thomson, M. De Noronha, and S. Joseph, "Are virtual reality technologies effective in improving lower limb outcomes for patients following stroke - a systematic review with metaanalysis," Topics in Stroke Rehabilitation, vol. 23, no. 6, pp. 440-457, 2016.

[2] I. J. M. de Rooij, I. G. L. van de Port, and J.-W. G. Meijer, "Effect of virtual reality training on balance and gait ability in patients with stroke: Systematic review and Meta-Analysis," Physical Therapy in Sport, vol. 96, no. 12, pp. 1905-1918, 2016.

[3] J. Iruthayarajah, A. McIntyre, A. Cotoi, S. Macaluso, and R. Teasell, "The use of virtual reality for balance among individuals with chronic stroke: A systematic review and meta-analysis," Topics in Stroke Rehabilitation, vol. 24, no. 1, pp. 68-79, 2017.

[4] M. C. Moreira, A. M. D. A. Lima, K. M. Ferraz, and M. A. B. Rodrigues, "Use of virtual reality in gait recovery among post stroke patients-a systematic literature review," Disability and Rehabilitation: Assistive Technology, vol. 8, no. 5, pp. 357-362, 2013.

[5] P. L. Weiss, R. Kizony, U. Feintuch, and N. Katz, "Virtual reality in neurorehabilitation," Textbook of Neural Repair and Rehabilitation, vol. 51, no. 8, pp. 182-197, 2006.

[6] L. Chen, W. L. A. Lo, Y. R. Mao et al., "Effect of virtual reality on postural and balance control in patients with stroke: a systematic literature review," BioMed Research International, vol. 2016, Article ID 7309272, 8 pages, 2016.

[7] S. Hwang, Understanding of Meta Analysis, Hakjisa, Seoul, Republic of Korea, 2015.

[8] B. J. Shea, L. M. Bouter, J. Peterson et al., "External validation of a measurement tool to assess systematic reviews (AMSTAR)," PLoS ONE, vol. 2, no. 12, Article ID el350, 2007.

[9] J. A. C. Sterne, A. J. Sutton, J. P. A. Ioannidis et al., "Recommendations for examining and interpreting funnel plot asymmetry in meta-analyses of randomised controlled trials," British Medical Journal, vol. 343, no. 7818, Article ID d4002, 2011.

[10] Y.-S. Bang, K. H. Son, and H. J. Kim, "Effects of virtual reality training using nintendo wii and treadmill walking exercise on balance and walking for stroke patients," Journal of Physical Therapy Science, vol. 28, no. 11, pp. 3112-3115, 2016.

[11] R. S. Calabrò, A. Naro, M. Russo et al., "The role of virtual reality in improving motor performance as revealed by EEG: a randomized clinical trial," Journal of NeuroEngineering and Rehabilitation, vol. 14, no. 1, 2017.

[12] K. H. Cho, K. J. Lee, and C. H. Song, "Virtual-reality balance training with a video-game system improves dynamic balance in chronic stroke patients," The Tohoku Journal of Experimental Medicine, vol. 228, no. 1, pp. 69-74, 2012.

[13] K. H. Cho and W. H. Lee, "Effect of treadmill training based real-world video recording on balance and gait in chronic stroke patients: a randomized controlled trial," Gait \& Posture, vol. 39, no. 1, pp. 523-528, 2014.

[14] N. M. Da Silva Ribeiro, D. Dominguez Ferraz, É. Pedreira et al., "Virtual rehabilitation via Nintendo Wii(R) and conventional physical therapy effectively treat post-stroke hemiparetic patients," Topics in Stroke Rehabilitation, vol. 22, no. 4, pp. 299305, 2015.

[15] G. G. Fluet, A. S. Merians, Q. Qiu, M. Rohafaza, A. M. Van Wingerden, and S. V. Adamovich, "Does training with traditionally presented and virtually simulated tasks elicit differing changes in object interaction kinematics in persons with upper extremity hemiparesis?" Topics in Stroke Rehabilitation, vol. 22, no. 3, pp. 176-184, 2015.

[16] T. In, K. Lee, and C. Song, "Virtual reality reflection therapy improves balance and gait in patients with chronic stroke: Randomized controlled trials," Medical Science Monitor, vol. 22, pp. 4046-4053, 2016.

[17] H. J. Sung, S. H. You, M. Hallett et al., "Cortical reorganization and associated functional motor recovery after virtual reality in patients with chronic stroke: an experimenter-blind preliminary study," Archives of Physical Medicine and Rehabilitation, vol. 86, no. 11, pp. 2218-2223, 2005.

[18] N. Kim, Y. Park, and B. H. Lee, "Effects of community-based virtual reality treadmill training on balance ability in patients with chronic stroke," Journal of Physical Therapy Science, vol. 27, no. 3, pp. 655-658, 2015.

[19] P. Kiper, M. Agostini, C. Luque-Moreno, P. Tonin, and A. Turolla, "Reinforced feedback in virtual environment for rehabilitation of upper extremity dysfunction after stroke: preliminary data from a randomized controlled trial," BioMed Research International, vol. 2014, Article ID 752128, 8 pages, 2014.

[20] D. Lee, M. Lee, K. Lee, and C. Song, "Asymmetric training using virtual reality reflection equipment and the enhancement of upper limb function in stroke patients: A randomized controlled trial," Journal of Stroke and Cerebrovascular Diseases, vol. 23, no. 6, pp. 1319-1326, 2014.

[21] H. Y. Lee, Y. L. Kim, and S. M. Lee, "Effects of virtual reality-based training and task-oriented training on balance performance in stroke patients," Journal of Physical Therapy Science, vol. 27, no. 6, pp. 1883-1888, 2015.

[22] S. Lee, Y. Kim, and B.-H. Lee, "Effect of virtual realitybased bilateral upper extremity training on upper extremity function after stroke: a randomized controlled clinical trial," Occupational Therapy International, vol. 23, no. 4, pp. 357-368, 2016. 
[23] R. Lloréns, J.-A. Gil-Gómez, M. Alcañiz, C. Colomer, and E. Noé, "Improvement in balance using a virtual realitybased stepping exercise: a randomized controlled trial involving individuals with chronic stroke," Clinical Rehabilitation, vol. 29, no. 3, pp. 261-268, 2015.

[24] J.-H. Park and J.-H. Park, "The effects of game-based virtual reality movement therapy plus mental practice on upper extremity function in chronic stroke patients with hemiparesis: A randomized controlled trial," Journal of Physical Therapy Science, vol. 28, no. 3, pp. 811-815, 2016.

[25] D.-S. Park, D.-G. Lee, K. Lee, and G. Lee, "Effects of virtual reality training using xbox kinect on motor function in stroke survivors: a preliminary study," Journal of Stroke and Cerebrovascular Diseases, vol. 26, no. 10, pp. 2313-2319, 2017.

[26] H. Sin and G. Lee, "Additional virtual reality training using Xbox kinect in stroke survivors with hemiplegia," American Journal of Physical Medicine \& Rehabilitation, vol. 92, no. 10, pp. 871-880, 2013.

[27] D. K. A. Singh, N. A. Mohd Nordin, N. A. A. Aziz, B. K. Lim, and L. C. Soh, "Effects of substituting a portion of standard physiotherapy time with virtual reality games among community-dwelling stroke survivors," BMC Neurology, vol. 13, article 199, 2013.

[28] G. b. Song and E. c. Park, "Effect of virtual reality games on stroke patients' balance, gait, depression, and interpersonal relationships," Journal of Physical Therapy Science, vol. 27, no. 7, pp. 2057-2060, 2015.

[29] R. T. Viana, G. E. C. Laurentino, R. J. P. Souza et al., "Effects of the addition of transcranial direct current stimulation to virtual reality therapy after stroke: a pilot randomized controlled trial," NeuroRehabilitation, vol. 34, no. 3, pp. 437-446, 2014.

[30] C. Yom, H.-Y. Cho, and B. H. Lee, "Effects of virtual realitybased ankle exercise on the dynamic balance, muscle tone, and gait of stroke patients," Journal of Physical Therapy Science, vol. 27, no. 3, pp. 845-849, 2015

[31] A. P. Verhagen, H. C. W. De Vet, R. A. De Bie et al., "The Delphi list: a criteria list for quality assessment of randomized clinical trials for conducting systematic reviews developed by Delphi consensus," Journal of Clinical Epidemiology, vol. 51, no. 12, pp. 1235-1241, 1998.

[32] N. C. Foley, R. W. Teasell, S. K. Bhogal, and M. R. Speechley, "Stroke rehabilitation evidence-based review: methodology," Topics in Stroke Rehabilitation, vol. 10, no. 1, pp. 1-7, 2003.

[33] J. Cohen, Statistical Power Analysis for The Behavioral Sciences, vol. 2, Hillsdale, NJ, USA, 1988.

[34] L. V. Hedges and I. Olkin, Statistical Methods for Meta-Analysis, Academic Press, Orlando, Fl, USA, 1985.

[35] R. Bernard and E. Borokhovski, Effect Size Calculation for MetaAnalysis, Campbell Colloquium, Oslo, Norway, 2009.

[36] C. B. Begg and M. Mazumdar, "Operating characteristics of a rank correlation test for publication bias," Biometrics, vol. 50, no. 4, pp. 1088-1101, 1994.

[37] M. Egger, G. D. Smith, M. Schneider, and C. Minder, "Bias in meta-analysis detected by a simple, graphical test," British Medical Journal, vol. 315, pp. 629-634, 1997.

[38] A. M. Tinga, J. M. A. Visser-Meily, M. J. van der Smagt, S. van der Stigchel, R. van Ee, and T. C. W. Nijboer, "Multisensory stimulation to improve low- and higher-level sensory deficits after stroke: a systematic review," Neuropsychology Review, vol. 26, no. 1, pp. 73-91, 2016.
[39] A. Aminov, J. M. Rogers, S. Middleton, K. Caeyenberghs, and P. H. Wilson, "What do randomized controlled trials say about virtual rehabilitation in stroke? A systematic literature review and meta-analysis of upper-limb and cognitive outcomes," Journal of NeuroEngineering and Rehabilitation, vol. 15, no. 1, 2018.

[40] Z. Li, X.-G. Han, J. Sheng, and S.-J. Ma, "Virtual reality for improving balance in patients after stroke: A systematic review and meta-analysis," Clinical Rehabilitation, vol. 30, no. 5, pp. 432-440, 2015.

[41] N. Takeuchi, T. Mori, Y. Suzukamo, and S.-I. Izumi, "Modulation of excitability in the temporoparietal junction relieves virtual reality sickness," Cyberpsychology, Behavior, and Social Networking, vol. 21, no. 6, pp. 381-387, 2018.

[42] K. E. Laver, B. Lange, S. George, J. E. Deutsch, G. Saposnik, and M. Crotty, "Virtual reality for stroke rehabilitation," Cochrane Database of Systematic Reviews, vol. 11, p. CD008349, 2017.

[43] G. Lee, "Effects of training using video games on the muscle strength, muscle tone, and activities of daily living of chronic stroke patients," Journal of Physical Therapy Science, vol. 25, no. 5, pp. 595-597, 2013.

[44] W. J. Kraemer, L. P. Koziris, N. A. Ratamess et al., "Detraining produces minimal changes in physical performance and hormonal variables in recreationally strength-trained men," The Journal of Strength and Conditioning Research, vol. 16, no. 3, pp. 373-382, 2002. 


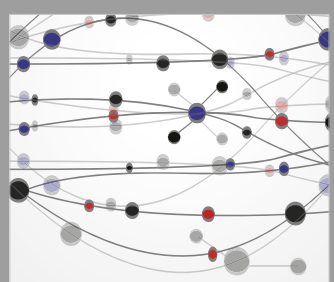

The Scientific World Journal
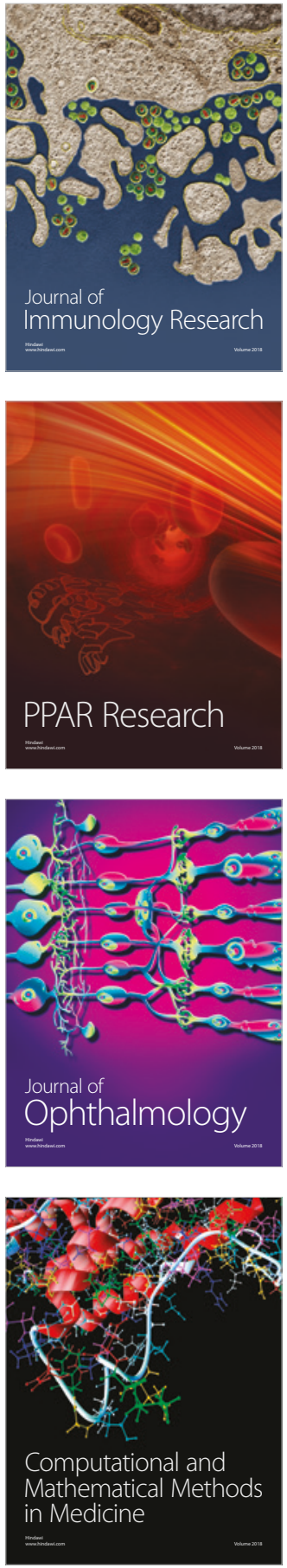

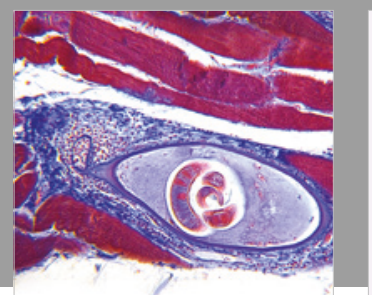

Gastroenterology Research and Practice

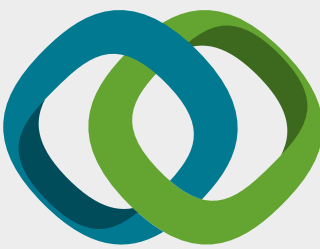

\section{Hindawi}

Submit your manuscripts at

www.hindawi.com
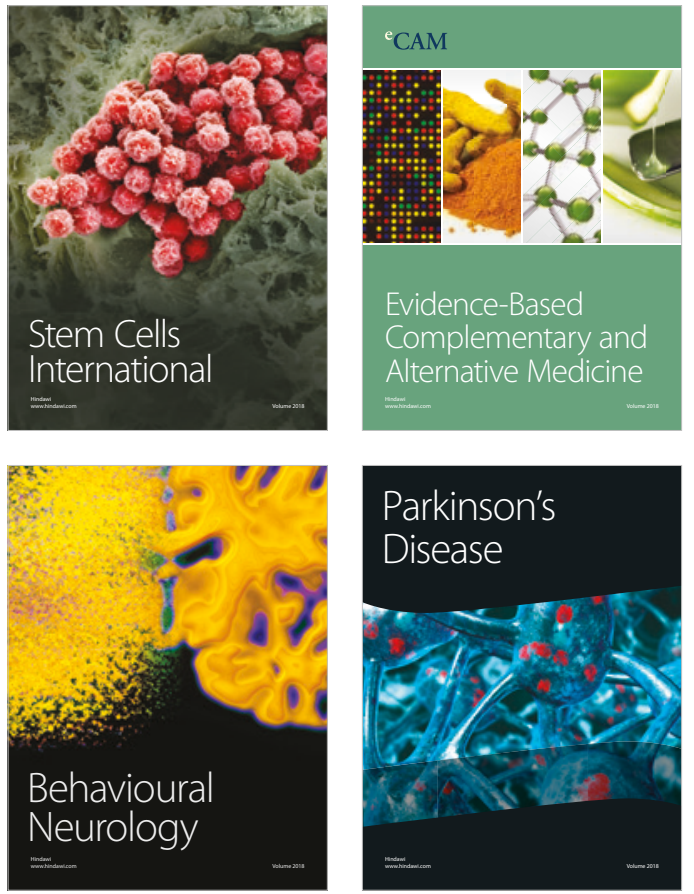

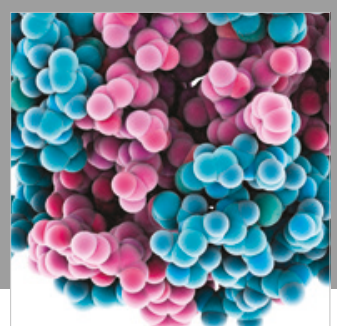

ournal of

Diabetes Research

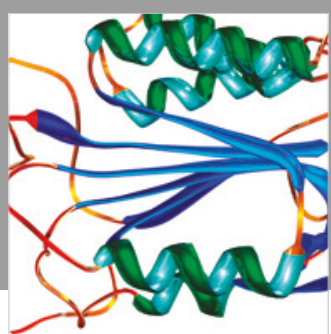

Disease Markers
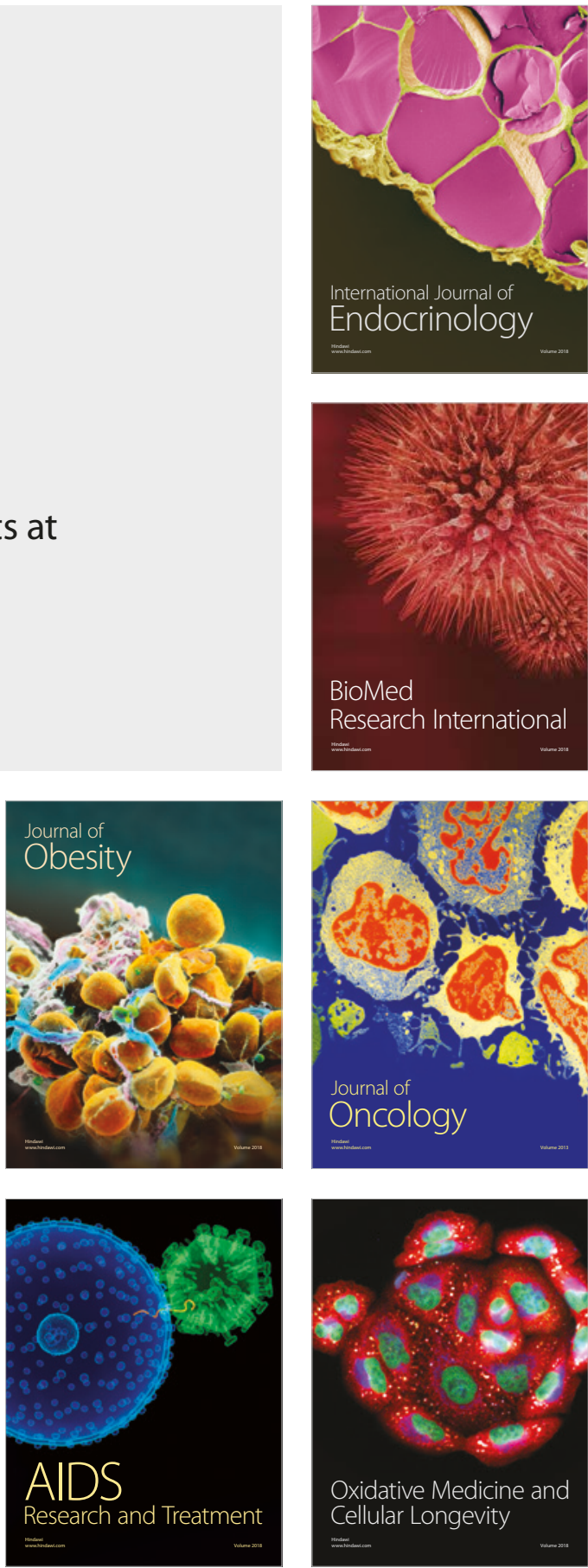